\title{
Perpendicular Magnetic Properties of [CoPt/AZO/Ag] Multilayered Films for Magneto-optical Chemical Sensing Applications
}

\author{
Haruki Yamane $^{1 *}$, Yuji Kondo ${ }^{1}$, Yasuyoshi Isaji ${ }^{2}$, Keisuke Takeda $^{2}$ and Masanobu Kobayashi ${ }^{2}$ \\ 1 Akita Industrial Technology Center, 4-21 Sanuki, Araya, Akita 010-1623, Japan \\ 2 Chiba Institute of Technology, 2-17-1 Tsudanuma, Narashino, Chiba 275-0016, Japan \\ * Corresponding author: Fax: 81-18-866-5803, e-mail: yamane@rdc.pref.akita.jp
}

\begin{abstract}
The perpendicular magnetic properties of $[\mathrm{CoPt} / \mathrm{AZO} / \mathrm{Ag}]$ multilayered films were investigated under polar Kerr measurement conditions. The magnetic properties were influenced by AZO layers formed upon the top and bottom surfaces of CoPt magnetic layer. The insertion of AZO intermediate layer into the $\mathrm{CoPt} / \mathrm{Ag}$ interface improved the perpendicular magnetic properties, and it was attributed to the improvement of the CoPt crystallinity. In contrast, the AZO overcoat layer which was directly formed on the top of the CoPt layer reduced the perpendicular magnetic anisotropy. This magnetic deterioration was suppressed by the oxidation of the CoPt surface. A plasma oxidation process provided good perpendicular magnetic properties with an ideal square out-of-plane Kerr loop.
\end{abstract}

Key words: perpendicular magnetic, magneto-optical, CoPt, multilayered film, chemical sensor

\section{INTRODUCTION}

The improvement of the magneto-optical (MO) Faraday and Kerr effects caused by magnetophotonic crystals (MPCs) and surface plasmon resonances (SPRs) has been extensively studied. Large MO activities are desirable in practical applications such as optical isolators, MO recording media, MO spatial light modulators, and MO chemical and biological sensors. Magnetic multilayered structures are a useful system for the improvement of MO activities. It has been reported that one-dimensional MPCs based on magnetic and nonmagnetic dielectric multilayers realize a huge Faraday rotation angle [1-5]. This MO enhancement originates from the localization of light at selected wavelengths in photonic band-gap structures. Similarly, optical cavities consisting of stacked layer structure enhance the MO Kerr effect by multiple reflections [610]. In recent years, it has been reported that the MO cavity indicates a very large MO enhancement factor reaching to two hundreds [11]. In addition to these MPCs, magneto-plasmonic effect on the layered structures consisting of magnetic transaction and noble metals has recently attracted considerable attention also [12-15]. A large MO enhancement caused by SPRs has been obtained in total reflection geometry using the Kretschmann-Raether configuration [16]. The MO amplification strongly depends on the plasmon excitation conditions at the surface of the magnetic multilayers. Therefore, it can be exploited as a new type of probe for chemical and biological sensors. Modulation techniques based on the combination of the MO effect and SPR have yielded high sensitivity [1720]. In addition to large MO effect, perpendicular magnetics is a prerequisite for many applications including the chemical and biological sensors. The polar Kerr system based on a normal incidence illumination does not require a prism coupler for the KretschmannRaether configuration. Surface sensitivity under the polar Kerr measurement system can propose a highly sensitive probe at a simple device configuration.

On this basis, we have investigated the magnetic and MO properties of Co-Pt alloys, multilayers and nanostructures [21-23]. The $\mathrm{Co}_{80} \mathrm{Pt}_{20}$-based films are used as high-density perpendicular magnetic recording media in commercial products. The CoPt film is a candidate material for many applications because of their large MO effect, perpendicular magnetic anisotropy and good chemical stability. In this study, we investigated the perpendicular magnetic properties of $[\mathrm{CoPt} / \mathrm{AZO} / \mathrm{Ag}]$ multilayered films for $\mathrm{MO}$ chemical sensing applications.

\section{EXPERIMENTS}

Samples were fabricated by magnetron sputtering onto a glass or thermal oxidized $\mathrm{Si}$ substrate with a 10-nm-thick Al-doped $\mathrm{ZnO}$ (AZO) seed layer. The base pressure of the sputtering system was about $7 \times 10^{-6} \mathrm{~Pa}$, and the Ar pressure was $0.2 \mathrm{~Pa}$ during the deposition. The AZO layers were fabricated by DC sputtering a $\mathrm{ZnO}$ target containing 0.06 wt. $\% \mathrm{Al}_{2} \mathrm{O}_{3}$. A 100 -nm-thick $\mathrm{Ag}$ was formed as a reflective layer, and it indicated a crystalline face-centered cubic (fcc)-(111) orientation along the surface normal by the AZO seed layer. The $\mathrm{CoPt}$ layer with crystalline hexagonal close-packed (hcp)-(001) orientation was fabricated by sputtering the Co-Pt composite target (80:20 at.\%), and the thickness was set to 3 or $5 \mathrm{~nm}$. The AZO films were also used as an intermediate layer at the $\mathrm{CoPt} / \mathrm{Ag}$ interface and an overcoat layer for the CoPt layer. The thicknesses of the AZO intermediate and overcoat layers were changed from $0 \mathrm{~nm}$ to $60 \mathrm{~nm}$ and from $0 \mathrm{~nm}$ to $30 \mathrm{~nm}$, respectively. The oxidation treatments for the surface of CoPt layer were performed by using air exposure and oxygen plasma. For the air exposure process, the sample was kept in the atmosphere for about 5 hours at ambient temperature. The plasma oxidation process was 
performed at the condition of $\mathrm{O}_{2}$ pressure of $1.5 \mathrm{~Pa}$ and $\mathrm{RF}$ power of $200 \mathrm{~W}$. The MO spectra were measured on a polar Kerr configuration in the wavelength range of 250-900 nm. A polarized incident light was irradiated along the surface normal of the samples. The film structure was measured by an out-of-plane and in-plane X-ray diffractometers (XRDs). The chemical conditions were nondestructively analyzed by using a hard X-ray photoelectron spectroscopy (XPS). The photon energy and proving depth for the hard XPS measurements were $7940 \mathrm{eV}$ and 5-20 $\mathrm{nm}$, respectively.

\section{RESULTS AND DISCUSSION}

\subsection{MO cavity effect}

Figures 1(a) and 1(b) show the polar Kerr spectra and hysteresis loops of the $\operatorname{CoPt}(5 \mathrm{~nm}) / \operatorname{Ag}(100 \mathrm{~nm})$ stacked films with and without a 60 -nm-thick AZO intermediate layer, respectively. The sign of Kerr rotation angle $\left(\theta_{\mathrm{K}}\right)$ was sharply reversed at the resonance wavelength $\left(\lambda_{R}\right)$ by inserting the AZO intermediate layer. A large Kerr angle of approximately 20 degrees was obtained at near the resonance wavelength. The layered structure is simple, but we can obtain a large $\mathrm{MO}$ enhancement factor of approximately 200. This MO enhancement is attributed to an optical interference (cavity) effect on the AZO intermediate layer. The sample is formed by a tri-layered structure consisting of magnetic surface half mirror, optical cavity and total reflection mirror, i.e. it is a Fabry-Pérot etalon. Figure 2(a) shows the schematic illustration for this $\mathrm{MO}$ cavity effect. The reflective light of low-order is canceled out when the optical thickness of the AZO cavity nearly equals to the quarter of the incident light wavelength. In this case, we can observe high-order reflective light only. This high-order light has large MO activities by multiple Kerr effects. Therefore, the Kerr rotation angle is related to the optical reflectivity $(R)$ of the sample. Figure 2(b) shows the

(a)

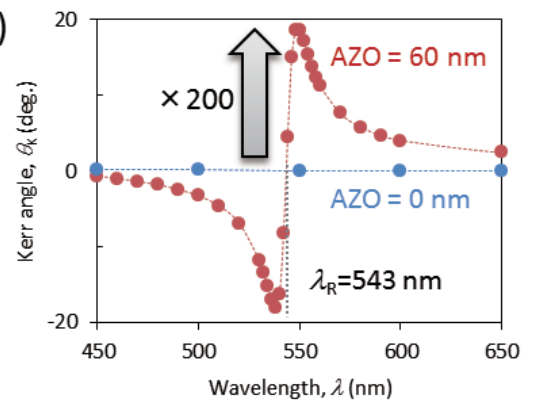

(b)

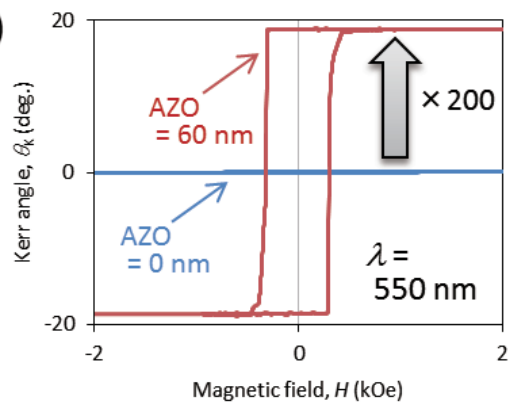

Fig. 1. (a) Polar Kerr spectra and (b) MO hysteresis loops for $[\operatorname{CoPt}(5 \mathrm{~nm}) / \operatorname{Ag}(100 \mathrm{~nm})]$ stacked films with and without $60-\mathrm{nm}$-thick AZO intermediate layer. optical simulation for the reflectivity spectra of the $[\mathrm{CoPt}(z \mathrm{~nm}) / \mathrm{AZO}(60 \mathrm{~nm}) / \mathrm{Ag}(100 \mathrm{~nm})]$ tri-layered films. The reflectivity sharply decreased at the resonance wavelength. The cavity effect can be controlled by carefully designing the thickness of each layer in the stacked structures. The resonance wavelength is mainly controlled by the thickness of the AZO cavity layer. The value of reflectivity strongly depends on the CoPt surface layer, and so the maximum Kerr angle is markedly changed by the condition of the magnetic surface layer. Therefore, the MO cavity can be used as an optical chemical sensor because the MO properties are sensitive to the optical conditions of the surface of the stacked films. A typical type of chemical sensing element consists of a surface detective, MO cavity and reflective mirror layers. We recently proposed a new type of hydrogen $\left(\mathrm{H}_{2}\right)$ gas sensor consisting of a $[\mathrm{Pd} / \mathrm{AZO} / \mathrm{CoPt} / \mathrm{AZO} / \mathrm{Pd}]$ stacked film [24]. In this case, the Pd surface thin film and [AZO/CoPt/AZO] tri-layer acted as a $\mathrm{H}_{2}$ reactive and $\mathrm{MO}$ cavity layers, respectively. We can provide various types of chemical sensor by changing the surface detective layer.

3.2 Effect of AZO intermediate layer

The MO chemical sensor requires not only large MO activity but also good perpendicular magnetic properties. The magnetic properties are important both for the stability on the sensing process and the electric driving power. The AZO intermediate layer was effective to improve also the perpendicular magnetic properties. Figure 3 shows the polar Kerr properties of $\operatorname{CoPt}(5$ $\mathrm{nm}) / \operatorname{Ag}(100 \mathrm{~nm})$ stacked films with various thicknesses of the AZO intermediate layer. The ratio of residual and saturation Kerr rotation angles $\left(\theta_{\mathrm{R}} / \theta_{\mathrm{S}}\right)$ markedly increased by inserting an only 2-nm-thick AZO layer. The $[\mathrm{CoPt} / \mathrm{AZO} / \mathrm{Ag}]$ stacked films indicated an ideal square polar Kerr loop with a low saturation magnetic field. It is known that the perpendicular magnetic anisotropy of the hcp(001) oriented CoPt film depends

(a)

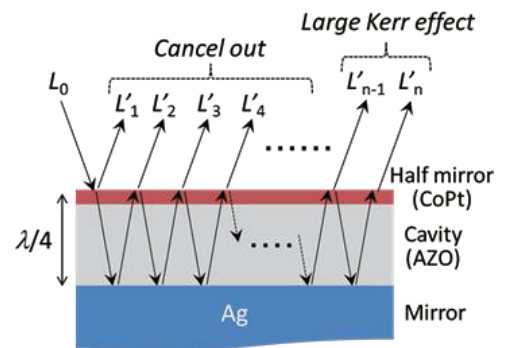

(b)

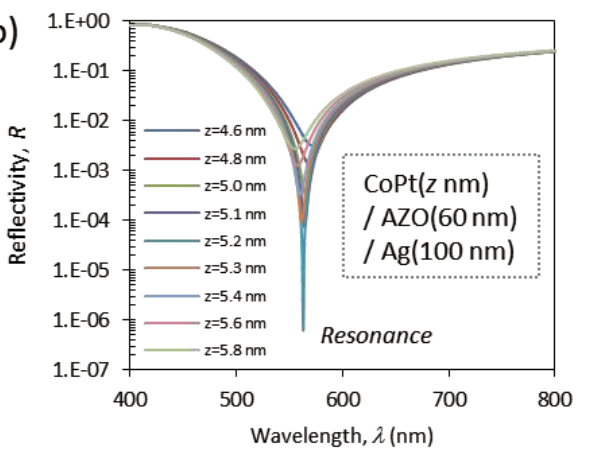

Fig. 2. (a) Schematic illustration of MO cavity effect and (b) optical simulation of reflectivity spectra for [CoPt/AZO/Ag] tri-layered films. 
on the crystalline quality. The fluctuation of the crystalline orientation reduces the magnetocrystalline anisotropy. Figures 4(a) and 4(b) show the out-of-plane and in-plane XRD measurements for $[\mathrm{CoPt}(5$ $\mathrm{nm}) / \mathrm{AZO}(0$ or $2 \mathrm{~nm}) / \operatorname{Ag}(100 \mathrm{~nm})]$ stacked films, respectively. The out-of-plane profiles of both samples indicate a single peak from the CoPt layer around $2 \theta=$ 43 degrees, and it is due to the diffraction from the $\mathrm{hcp}(002)$ and/or fcc(111) planes. There was no evidence to support the improvement of the crystalline quality of the CoPt layer in the out-of-plane XRD measurement. On the other hand, the in-plane XRD profiles suggested that the CoPt crystallinity was improved by inserting the AZO intermediate layer. As shown in Fig. 4(b), two diffractions from the CoPt layer are observed at low and high angle regions. These peaks are identified as the diffractions from the $h c p(100)$ plane and from the hcp(110) and/or fcc(220) planes. S. Saito et al. reported

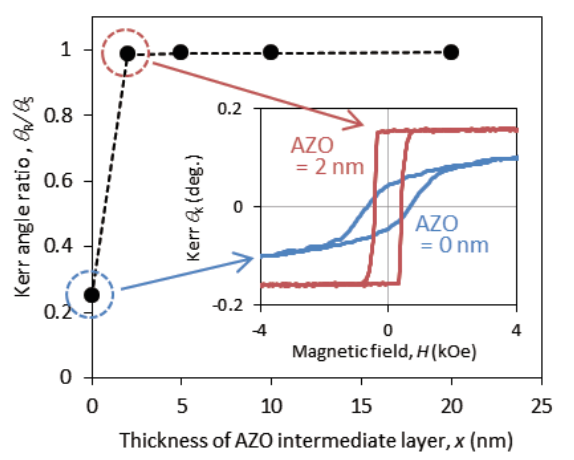

Fig. 3. Ratio of residual and saturation Kerr rotation angles $\left(\theta_{\mathrm{R}} / \theta_{\mathrm{S}}\right)$ of $[\operatorname{CoPt}(5 \mathrm{~nm}) / \operatorname{Ag}(100$ $\mathrm{nm})$ ] stacked films with various thicknesses of AZO intermediate layer.

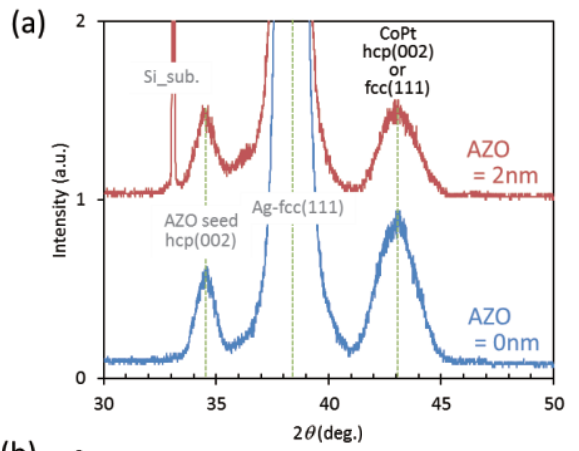

(b)

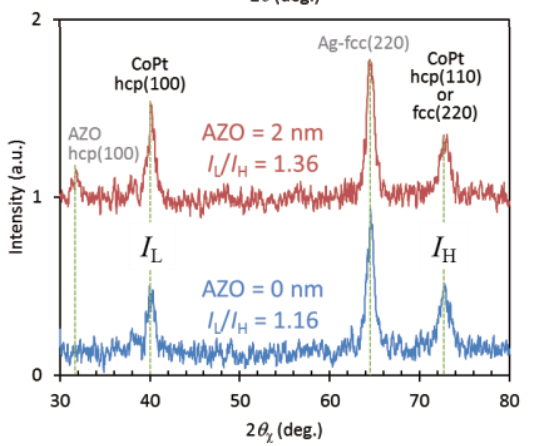

Fig. 4. (a) Out-of-plane and (b) in-plane XRD profiles for $\mathrm{CoPt} / \mathrm{Ag}$ stacked films with and without 2-nm-thick AZO intermediate layer. that the ratio of the intensities of these peaks can be used as an index for atomic stacking faults along the surface normal in the hcp $(001)$ oriented films [25]. The ratio of the integrated intensities $\left(I_{\mathrm{L}} / I_{\mathrm{H}}\right)$ was increased from 1.16 to 1.36 by inserting the $\mathrm{AZO}$ layer into the $\mathrm{CoPt} / \mathrm{Ag}$ interface, i.e. the AZO intermediate layer reduced the content of atomic stacking faults in the CoPt layer. The improvement of the perpendicular magnetic properties is attributed to the increasing of the magnetocrystalline anisotropy. Moreover, we investigated also the influence of the AZO intermediate layer on the chemical condition of the CoPt/Ag stacked films by using nondestructive XPS measurements. The hard XPS spectra indicated that the chemical conditions of $\mathrm{Co}, \mathrm{Pt}$ and $\mathrm{Ag}$ were not affected by inserting the AZO intermediate layer.

3.3 Effect of AZO overcoat layer

The perpendicular magnetic properties of the $\mathrm{CoPt}$ stacked films were also influenced by an AZO overcoat layer formed on the surface of the $\mathrm{CoPt}$ layer. Figure 5(a) shows the $\theta_{\mathrm{R}} / \theta_{\mathrm{S}}$ of the $[\mathrm{CoPt} / \mathrm{AZO} / \mathrm{Ag}]$ stacked films with various thicknesses of AZO overcoat layer. The sample structure is $[\mathrm{AZO}(x \mathrm{~nm}) / \operatorname{CoPt}(5 \mathrm{~nm}) / \mathrm{AZO}(2$ $\mathrm{nm}) / \mathrm{Ag}(100 \mathrm{~nm})]$. Blue circle indicates the result for the samples with the AZO overcoat layer which was directly deposited onto the CoPt surface. The AZO overcoat layer reduced the perpendicular magnetic properties. This magnetic deterioration can be suppressed by air exposure (red circle) for the surface of CoPt layer. The samples were kept in the atmosphere for about 5 hours before the AZO overcoat layer was deposited on the CoPt layer. As shown in Fig. 5(b), the air exposure sample indicated good perpendicular magnetic properties with $\theta_{\mathrm{R}} / \theta_{\mathrm{S}}=1$. The hard XPS measurements suggested that this phenomenon was due to the difference in the surface oxidization of the CoPt layer. Figure 6 shows the nondestructive XPS profiles for Co

(a)

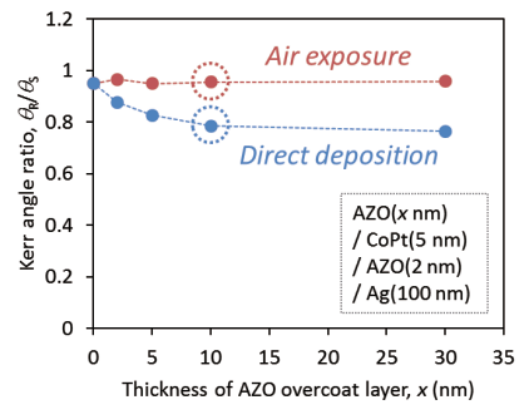

(b)

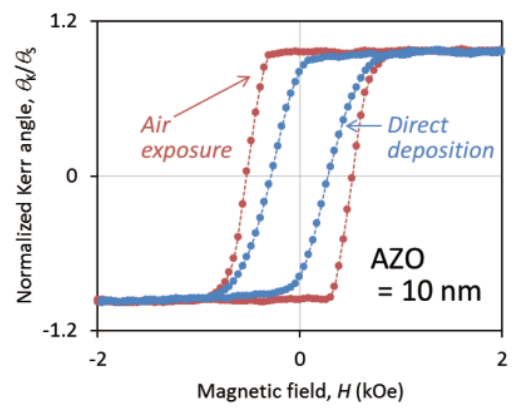

Fig. 5. (a) Kerr angle ratio $\left(\theta_{\mathrm{R}} / \theta_{\mathrm{S}}\right)$ of $[\mathrm{CoPt}(5$ $\mathrm{nm}) / \operatorname{AZO}(2 \mathrm{~nm}) / \operatorname{Ag}(100 \mathrm{~nm})]$ stacked films with various thicknesses of AZO overcoat layer. (b) Polar Kerr loops of the samples with and without air exposure process. 
and $\mathrm{Zn}$. The air exposure process promoted the oxidization of $\mathrm{Co}$ at the interface between the CoPt and AZO overcoat layers. The binding energies of Co- $2 p_{3 / 2}$ at the metal and oxide states are $778.2 \mathrm{eV}$ and $779.7 \mathrm{eV}$, respectively. The oxidation state of $\mathrm{Co}$ can be evaluated from these XPS peaks. The intensity ratio of the

(a)

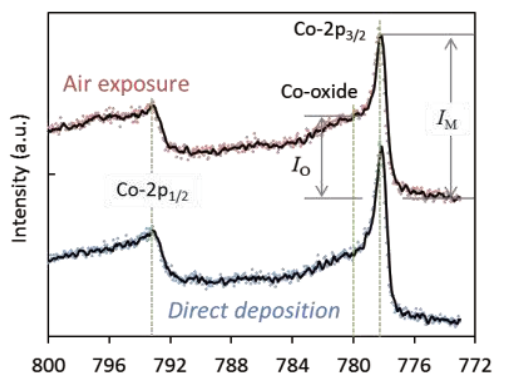

(b)

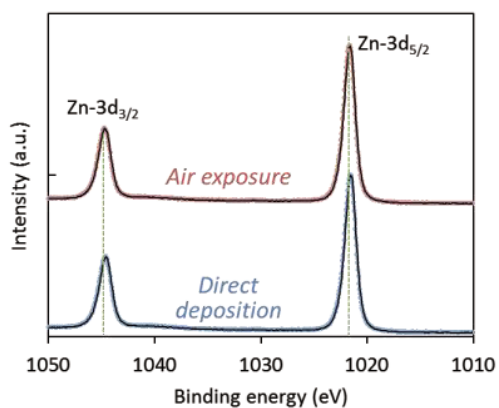

Fig. 6. XPS profiles of (a) Co and (b) $\mathrm{Zn}$ for $[\mathrm{AZO}(10 \mathrm{~nm}) / \operatorname{CoPt}(3 \mathrm{~nm}) / \operatorname{AZO}(2 \mathrm{~nm}) / \operatorname{Ag}(100$ $\mathrm{nm})$ ] stacked films with and without air exposure process for $\mathrm{CoPt}$ surface.

(a)

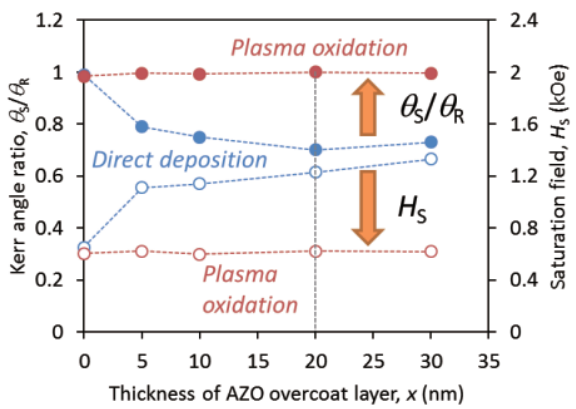

(b)

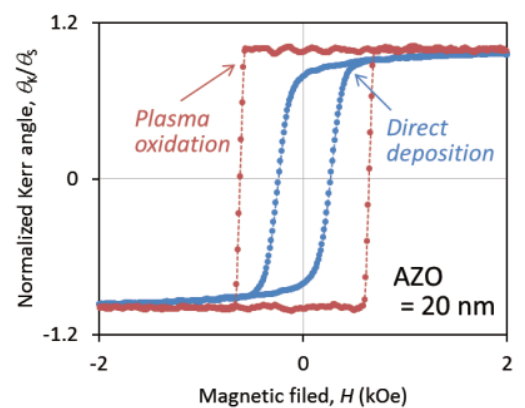

Fig. 7. (a) Influence of AZO overcoat layer on perpendicular magnetic properties $\left(\theta_{\mathrm{R}} / \theta_{\mathrm{S}} \&\right.$ $\left.H_{\mathrm{S}}\right)$ of $[\mathrm{CoPt}(3 \mathrm{~nm}) / \operatorname{AZO}(2 \mathrm{~nm}) / \operatorname{Ag}(100 \mathrm{~nm}]$ stacked films. (b) Polar Kerr loops of the samples with and without plasma oxidation process for 60 seconds.
Co- $2 \mathrm{p}_{3 / 2}$ oxide and metal peaks $\left(I_{\mathrm{O}} / I_{\mathrm{M}}\right)$ increased from 0.41 to 0.51 by the air exposure. Recently, D. Chiba et al reported that the magnetic anisotropy of Co ultra-thin films on a $\mathrm{ZnO}$ single-crystal substrate exhibited drastic polarity-dependent differences in the $\mathrm{Zn}$ - and O-polar surfaces of the substrate [26]. The Co film formed on the Zn-polar surface indicated in-plane magnetic properties, whereas the Co film on the O-polar surface had a perpendicular magnetic easy axis. In our case, the oxidation at the $\mathrm{AZO} / \mathrm{CoPt}$ interface also is important for the perpendicular magnetic properties. In the same way, the perpendicular magnetics can be improved by also plasma oxidation (P.O.) for the surface of CoPt layer. Figure 7 shows the $\theta_{\mathrm{R}} / \theta_{\mathrm{S}}$ and magnetic saturation field $\left(H_{\mathrm{S}}\right)$ of the $[\mathrm{AZO}(x \mathrm{~nm}) /(\mathrm{P} . \mathrm{O}.) / \operatorname{CoPt}(3 \mathrm{~nm}) / \mathrm{AZO}(2$ $\mathrm{nm}) / \operatorname{Ag}(100 \mathrm{~nm})]$ stacked films with and without the plasma oxidation process for 60 seconds. The direct deposition of the AZO overcoat layer (blue circle) reduced the perpendicular magnetic properties. In contrast, the plasma oxidation (red circle) provided good perpendicular magnetic properties with a low $H_{\mathrm{S}}$ and $\theta_{\mathrm{R}} / \theta_{\mathrm{S}}=1$. As shown in Fig. 7(b), the plasma oxidized $[\mathrm{AZO} / \mathrm{CoPt} / \mathrm{AZO} / \mathrm{Ag}]$ stacked films indicated an ideal square polar Kerr loop.

\section{CONCLUSION}

The perpendicular magnetic and polar Kerr properties of $[\mathrm{CoPt} / \mathrm{AZO} / \mathrm{Ag}]$ multilayered films were investigated. The AZO intermediate layer inserted into the $\mathrm{CoPt} / \mathrm{Ag}$ interface provided a large Kerr rotation angle due to the cavity effect. The MO enhancement factor reached to approximately 200. Moreover, the AZO intermediate layer improved also the perpendicular magnetic properties. The in-plane XRD measurements suggested that this magnetic improvement was due to the improvement of the crystalline quality of the CoPt layer. In contrast, the AZO overcoat layer formed on the $\mathrm{CoPt}$ layer decreased the perpendicular magnetic anisotropy. This magnetic deterioration was suppressed by the oxidation of the CoPt surface. This sample indicated an ideal square polar Kerr loop with low saturation magnetic field. The $[\mathrm{CoPt} / \mathrm{AZO} / \mathrm{Ag}]$ multilayered films will be suitable for the MO chemical sensing applications.

\section{Acknowledgment}

This study was partly supported by JSPS KAKENHI (Grant no. 17K05087). The hard X-ray photoelectron spectroscopy experiments were performed at SPring-8 (Proposal no. 2016A1226 / BL47XU).

\section{REFERENCES}

[1] M. Inoue, K. Arai, T. Fujii, and M. Abe, J. Appl. Phys. 85, 5768 (1999).

[2] M. J. Steel, M. Levy, and R. M. Osgood, IEEE Phot. Tech. Lett. 12, 1171 (2000).

[3] S. Kahl and A. M. Grishin, Appl. Phys. Lett. 84, 1438 (2004).

[4] M. Inoue, R. Fujikawa, A. Baryshev, A. Khanikaev, P. B. Lim, H. Uchida, O. Aktsipetrov, A. Fedyanin, T. Murzina, and A. Granovsky, J. Phys. D: Appl. Phys. 39, R151 (2006).

[5] L. Dong, H. Jiang, H. Chen, and Y. Shi, J. Appl. Phys. 107, 093101 (2010). 
[6] K. Ohta, A. Takahashi, T. Deguchi, T. Hyuga, S. Kobayashi, and H. Yamaoka, Proceedings of International Society for Optics and Photonics 0382, 252 (1983).

[7] K. Nakamura, T. Asaka, S. Asari. Y. Ota, and A. Ito, IEEE Trans. Magn. 21, 1654 (1985).

[8] Y. Wang, W. M. Zheng, S. M. Zhou, L. Y. Chen, W. R. Zhu, D. L. Qian, Y. X. Zheng, H. Y. Zhang, and J. Huang, J. Appl. Phys. 81, 1956 (1997).

[9] N. Qureshi, H. Schmidt, and A. R. Hawkins, Appl. Phys. Lett. 85, 431 (2004).

[10] S. Y. Zhang, J. L. Gao, W. B. Xia, X. J. Luo, S. L. Tang, and Y. W. Du, J. Appl. Phys. 114, 064308 (2013). [11] H. Yamane, K. Takeda and M. Kobayashi, Mater. Trans. 57, 892 (2016).

[12] V. I. Safarov, V. A. Kosobukin, C. Hermann, G. Lampel, J. Peretti, and C. Marliere, Phys. Rev. Lett. 73, 3584 (1994).

[13] C. Hermann, V. A. Kosobukin, G. Lampel, J. Peretti, V. I. Safarov, and P. Bertrand, Phys. Rev. B 64, 235422 (2001).

[14] J. B. González-Díaz, A. García-Martín, G. Armelles,J. M. García-Martín, C. Clavero, A. Cebollada, R. A. Lukaszew, J. R. Skuza, D. P. Kumah, and R. Clarke, Phys. Rev. B 76, 153402 (2007).

[15] H.-T. Huang, T.-R. Ger, Y.-R. Xu, C.-Y. Huang, K.-T. Liao, J.-Y. Lai, J.-Y. Chen, C.-H. Chen, and Z.-H. Wei, J. Appl. Phys. 115, 17E313 (2014).
[16] E. Kretschmann and H. Raether, Z. Naturforsch 23a, 2135 (1968).

[17] B. Sepúlveda, A. Calle, L. M. Lechuga, and G. Armelles, Opt. Lett. 31, 1085 (2006).

[18] D. Regatos, D. Farina, A. Calle, A. Cebollada, B. Sepulveda, G. Armelles, and L. M. Lechuga, J. Appl. Phys. 108, 054502 (2010).

[19] D. Regatos, B. Sepulveda, D. Farina, L. G. Carrascosa, and L. M. Lechuga, Opt. Express 19, 8336 (2011).

[20] M. G. Manera, A. Colombelli, R. Rella, A. Caricato, P. D. Cozzoli, M. Martino, and L. Vasanelli, J. Appl. Phys. 112, 053524 (2012).

[21] H. Yamane, Appl. Phys. Lett. 102, 072412 (2013)

[22] H. Yamane and M. Kobayashi, Jpn. J. Appl. Phys. 53, 05FB04 (2014).

[23] H. Yamane, K. Takeda and M. Kobayashi, Appl. Phys. Lett. 106, 052409 (2015).

[24] H. Yamane, H. Yamasaki, and K. Sumiyoshi, K. Shigemura, Digest of 29th International Microprocesses and Nanotechnology Conference (MNC2016), 10P-7-21 (2016).

[25] S. Saito, A. Hashimoto, D. Hasegawa, and M. Takahashi, J. Phys. D: Appl. Phys., 42, 145007-1-7 (2009).

[26] D. chiba, N. Shibata, and A. Tsukazaki, Scientific Rep., 6, 38005 (2016).

(Received November 7, 2017; Accepted May 11, 2018; Published Online August 1, 2018) 